The answers, as one would expect, vary a good deal from case to case, but the essential need for models of this sort to bridge the gap between purely in-vitro studies and application in man is convincingly demonstrated.

H. P. Lambert

\title{
The epidemiology of human mycotic disease
}

Edited by Yousef Al-Doory. 1975. Springfield, Illinois: Charles C. Thomas. Pp. xv and 346. $£ 14 \cdot 60$.

As Dr L. Ajello emphasises, on p. 290 of this book, the true incidence of mycotic disease and its socioeconomic impact are still unknown. Fungal diseases, particularly the cutaneous mycoses, are common and constitute a real public health hazard. The subcutaneous and deep-seated (systemic) mycoses, though less frequently encountered in Western countries, exact a considerable toll of lives and pose practical and fundamental problems for physician and pathologist alike.

The Editor has done a service to medicine by compiling the major epidemiological features of the mycoses of man in a single, readable and adequately referenced volume. There are few texts that deal generally with the mycoses, and few, indeed, that can serve pathologists both as a reference source and as refresher material. This book should prove useful to the tyro, no less than to the expert, and can be recommended to both.

Rosalinde HurLeY

Cell membrane receptors for viruses, antigens and antibodies, polypeptide hormones, and small molecules

Edited by R. F. BeERS, JR and E. G. BASSETT. 1976. Ninth Miles International Symposium. New York: Raven Press. Pp. xiv and 540. US \$43.50.

Published reports of meetings are generally either out of date by the time they appear on the market, or printed by cheap and rapid processes in such a manner that they come to resemble a PhD thesis or a florist's guide rather than a scientific tome. The Ninth Miles International Symposium on cell-membrane receptors is neither. Although the date of the symposium is carefully withheld from the reader, the number of references to papers published in 1975, and some even in 1976, testify to a rapid and efficient editorial process. Equally commendable are the layout and production of the book. Clear tables and figures, including some excellent electron micrographs, accompany each chapter. The latter word is used deliberately, for that is what the main presentations of the symposium have become: a clear summary of the current status of the field for expert and general reader alike, followed by a well-balanced account of the most recent results, fully but not inordinately referenced. A refreshing change from the type of chapter so often found in Annual Reviews.

The book is divided into four main sections. The first, on polypeptide-hormone receptors, covers recent work on the binding of insulin, growth hormone, prolactin, luteinising hormone and chorionic gonadotrophin. Not only is the isolation and function of the receptors described but also-exemplified by an interesting chapter on insulin receptors in disease by C. $\mathbf{R}$. Kahn-their physiological significance.

The second section, on virus receptors, deals with picornaviruses, adenoviruses, paramyxoviruses, RNA tumour-viruses and poxviruses. Cunningly inserted is a short summary on the " glycophorin" of red cells, by V. T. Marchesi, on the grounds that it is, among other things, the receptor for influenza virus.

The third section describes the receptors on $\mathbf{B}$ and $\mathbf{T}$ lymphocytes, the consequences of IgE binding and the rather complex macrophage-lymphocyte system. One wishes that this part had been a little longer.

The final section is on receptors for small molecules. It ranges from the acetylcholine 